\title{
Composición Factorial de la Escala de Autoeficacia Académica en Universitarios Mexicanos
}

\author{
Humberto Blanco, Juan F. Aguirre, Juan C. Barrón y José R. Blanco* \\ Universidad Autónoma de Chihuahua. Facultad de Ciencias de la Cultura Física. DES Salud CA 1121. \\ Calle Escorza 900, CP 31000 Chihuahua, Chih.-México (e-mail: hblanco@uach.mx, jaguirre@uach.mx, \\ jcbarron@uach.mx y jblanco@uach.mx)
}

${ }^{*}$ Autor a quien debe ir dirigida la correspondencia

Recibido Ago. 14, 2015; Aceptado Oct. 6, 2015; Versión final Oct. 17, 2015, Publicado Abr. 2016

\section{Resumen}

Se presenta un estudio que indaga si es posible replicar los resultados psicométricos propuestos por Rangel para la Escala de Autoeficacia Académica. La muestra total fue de 716 hombres alumnos de las licenciaturas de Educación Física y Motricidad Humana que se ofrecen en la Universidad Autónoma de Chihuahua en México con una edad media de 20.8 años. La estructura factorial del cuestionario se analizó a través de análisis factoriales confirmatorios. Los análisis, muestran que una estructura tetra factorial es viable y adecuada. La estructura de cuatro factores incluye comprensión, comunicación, atención y excelencia. Esta ha mostrado adecuados indicadores de ajuste de fiabilidad y validez. Además, los resultados de los análisis factoriales llevados a cabo con las submuestras, indican la existencia de fuertes evidencias de la estabilidad de la estructura factorial.

Palabras clave: autoeficacia, estructura factorial, validación de constructo, ecuaciones estructurales

\section{Factor Structure of the Self-Efficacy Scale in Mexican University Students}

\begin{abstract}
The present study investigates the psychometric results proposed by Rangel for the Academic Self-Efficacy Scale. The total sample included 716 male students, with a mean age of 20.8 years, of the degree of Physical Education and Human Motricity offered at the Autonomous University of Chihuahua in Mexico. Psychometric analysis showed that a tetra-factorial structure was viable and adequate. The factorial structure of the questionnaire was analyzed with confirmatory factor analysis. The four-factor structure included comprehension, communication, attention and excellence. This showed adequate indicators of adjustment of reliability and validity. In addition, factor analysis conducted with subsamples showed the presence of strong evidence of factor structure stability.
\end{abstract}

Keywords: self-efficacy, factor structure, constructs validation, structural equation 


\section{INTRODUCCIÓN}

La motivación y el aprendizaje en los estudiantes son dos de las preocupaciones más importantes de la mayoría de los profesores, donde las creencias de éstos acerca de sus habilidades para llevar a cabo sus tareas académicas constituyen una de las variables más importantes que influyen en el rendimiento académico; puesto que las personas pueden realizar mal en tareas no necesariamente porque carecen de la capacidad de tener éxito, sino porque carecen de confianza en sus capacidades (Hasheminasab et al., 2014; Robbins et al., 2004). Una forma de conceptualizar las creencias de los estudiantes sobre sus capacidades para llevar a cabo sus tareas escolares que ha sido utilizado por los investigadores de la motivación es la autoeficacia; se ha demostrado que niveles altos de autoeficacia conducen a un mejor rendimiento en tareas académicas (Bandura, 1997; Javanmard et al., 2012).

La autoeficacia se refiere a la creencia de que uno puede lograr los resultados deseados y es un constructo central en la teoría cognitiva social de Bandura $(1986,2012)$. De acuerdo con la teoría, la autoeficacia de un individuo es un factor fundamental en la interacción entre el medio ambiente y el comportamiento del individuo (Bandura, 2012). La autoeficacia puede ser específica o general. La autoeficacia específica describe las creencias de un individuo sobre el que él puede lograr buenos resultados en un área definida de su vida, por ejemplo su rendimiento académico. Mientras que la autoeficacia general es el sentido global de competencia de un individuo en el manejo de una variedad de desafíos de la vida. Ambos tipos de autoeficacia son relativamente estables y se pueden caracterizar como rasgos (Yeo y Neal, 2006).

Varios estudios (Bandura, 1997; Beaudoin y Desrichard, 2011; Hannah et al., 2012; Richardson et al., 2012; Robbins et al., 2004) han establecido, de manera amplia, que una alta autoeficacia académica está asociada con mejores resultados en lo que a rendimiento académico se refiere; poniendo de manifiesto que no basta con ser capaz de, es preciso juzgarse capaz. Dejando ver porque las personas con el mismo nivel de habilidad y conocimiento presentan conductas y/o resultados diferentes, o por qué las personas actúan en disonancia con sus habilidades (Bandura, 1982; Pérez et al., 2011). Evidenciando que las creencias de autoeficacia en la propia capacidad son imprescindibles para dominar las actividades académicas; ya que los estudiantes que confían en sus capacidades se sienten más motivados para alcanzar sus metas (Á. Blanco, 2010; Rodríguez, 2009).

Por todo lo antes dicho, esta investigación se basa en la premisa de que la autoeficacia académica percibida es un importante factor mediador en cómo la gente se siente, piensa, se motivan y se comporta; por lo que medir la percepción de autoeficacia académica en quien aprende es sumamente importante en el estudio de como facilitar el progreso y el éxito educativos, así como el de minimizar el riesgo de abandonar la escuela (Peguero y Shaffer, 2015; Shkullaku, 2013). Por ello el presente estudio instrumental (Montero y León, 2005) se ha dirigido a proporcionar apoyo empírico a la división factorial de la Escala de Autoeficacia Académica; lo que se justifica por la importancia de comprobar la estructura factorial de un instrumento y la equivalencia psicométrica del mismo en distintos grupos; ya que en el contexto de la comparación intergrupal, es indispensable plantearse la necesidad de llevar a cabo la adaptación de un instrumento de medida psicológica que cumpla con todos los criterios de equivalencia, pero sobre todo plantearse si la misma estructura factorial es aplicable a distintos grupos de sujetos 0 , de modo más genérico, a distintas poblaciones (Abalo et al., 2006).

\section{METODOLOGÍA}

\section{Participantes}

La muestra de 716 participantes se obtuvo mediante un muestreo por conveniencia, tratando de abarcar la representatividad de las diferentes licenciaturas que se ofrecen en la Facultad de Ciencias de la Cultura Física de la Universidad Autónoma de Chihuahua. La edad de los participantes fluctuó entre los 18 y 26 años, con una media de 20.78 y una desviación estándar de 1.94 años. La muestra fue aleatoriamente dividida en dos partes utilizando el Statistical Package for the Social Sciences (SPSS) en su versión 18.0 con el fin de realizar estudios paralelos que permitieran corroborar y verificar los resultados obtenidos (validación cruzada). La submuestra 1 quedo constituida por 357 hombres. Las edades fluctúan entre los 18 y 26 años, con una media de 20.78 y una desviación estándar de 1.87 años. La submuestra 2 quedo compuesta por 359 hombres. Las edades fluctúan entre los 18 y 26 años, con una media de 20.78 y una desviación estándar de 2.00 años.

\section{Instrumento}

Escala de Autoeficacia Académica, es una encuesta tipo Likert, asistida por computadora de 16 reactivos donde el encuestado responde, en una escala de 0 a 10, que tan capaz se siente en cada uno de los reactivos de los factores de la escala (Comprensión, Comunicación, Atención y Excelencia). 
De acuerdo al estudio de Rangel (2015) Los resultados globales del análisis factorial confirmatorio para la escala de autoeficacia académica (GFI .966; RMSEA .43; CFI .982) que corresponde a la estructura tetradimensional del cuestionario indican que su ajuste es óptimo. Los cuatro factores de este modelo alcanzan valores de consistencia interna por encima de .75 evidenciando una consistencia interna adecuada. Por otro todos los ítems saturan adecuadamente en su dimensión (factor) prevista con saturaciones de .70 o mayores. Observándose además, intercorrelaciones altas entre los cuatro factores evidenciando una no muy adecuada validez discriminante entre ellos.

\section{Procedimiento}

Se invitó a participar en el estudio a los alumnos de las licenciaturas que se ofrecen en la Facultad de Ciencias de la Cultura Física (FCCF) de la Universidad Autónoma de Chihuahua. Los que aceptaron participar firmaron la carta de aceptación correspondiente. Luego se aplicó el instrumento, antes descrito, por medio de una computadora personal (módulo administrador del instrumento del editor de escalas de ejecución típica), en una sesión de aproximadamente 25 minutos en los laboratorios o centros de cómputo de la FCCF. Al inicio de cada sesión se hizo una pequeña introducción sobre la importancia de la investigación y de cómo acceder al instrumento. Se les solicitó la máxima sinceridad y se les garantizó la confidencialidad de los datos que se obtuvieran. Las instrucciones de cómo responder se encontraban en las primeras pantallas antes del primer reactivo del instrumento. Al término de la sesión se les agradeció su participación. Una vez aplicado el instrumento se procedió a recopilar los resultados por medio del módulo generador de resultados del editor de escalas versión 2.0 (H. Blanco et al., 2013).

\section{Análisis de datos}

El primer paso del análisis de las propiedades psicométricas del cuestionario consistió en calcular la media, la desviaciones estándar, la asimetría, la curtosis y los índices de discriminación de cada ítem. Para luego eliminar de la escala aquellos que obtienen una curtosis o asimetría extremas, o un índice de discriminación por debajo de 0.30 (Brzoska y Razum, 2010). Luego, se sometieron a comparación dos modelos de medida: el Modelo 1 (M1), modelo de cuatro factores acorde a la distribución original de los reactivos dentro del cuestionario y el Modelo 2 (M1b), que responde a la estructura factorial del modelo anterior, eliminando los reactivos que no fueron suficientemente bien explicados por ese modelo y/o obtuvieron un índice de discriminación bajo.

Para conducir los análisis factoriales confirmatorios se utilizó el software AMOS 21 (Arbuckle, 2012), las varianzas de los términos de error fueron especificados como parámetros libres, en cada variable latente (factor) se fijó uno de los coeficientes estructurales asociados a uno, para que su escala sea igual a la de una de las variables observables (reactivos). El método de estimación empleado fue el de Máxima Verosimilitud siguiendo la recomendación de Thompson (2004), en el sentido de que cuando se emplea análisis factorial confirmatorio se debe corroborar no sólo el ajuste de un modelo teórico sino que es recomendable comparar los índices de ajuste de varios modelos alternativos para seleccionar el mejor.

Para evaluar el ajuste del modelo se emplearon el estadístico Chi-cuadrado, el índice de bondad de ajuste (GFI) y el error cuadrático medio de aproximación (RMSEA) como medidas absolutas de ajuste. El índice de bondad ajustado (AGFI), el Índice Tucker-Lewis (TLI) y el índice de bondad de ajuste comparativo (CFI) como medidas de ajuste incremental. La razón de Chi-cuadrado sobre los grados de libertad (CMIN/GL) y el Criterio de Información de Akaike (AIC) como medidas de ajuste de parsimonia (Byrne, 2010; Gelabert et al., 2011). Posteriormente, siguiendo las recomendaciones de Abalo et al. (2006), se llevó a cabo un análisis de la invarianza factorial del cuestionario para las submuestras tomando como base el mejor modelo de medida obtenido en la etapa anterior. Por último se calculó la fiabilidad de cada una de las dimensiones, de los modelos de medida obtenidos en cada submuestra, a través del Coeficiente Alpha de Cronbach (Elosua y Zumbo, 2008; Nunnally y Bernstein, 1995) y del Coeficiente Omega (Revelle y Zinbarg, 2009; Sijtsma, 2009).

\section{RESULTADOS}

\section{Análisis descriptivos e índices de discriminación}

En la Tabla 1 se resumen los resultados de los análisis descriptivos y los índices de discriminación (correlación elemento-total corregida) de cada uno de los 16 reactivos del cuestionario en la muestra total. Las respuestas a todos los reactivos reflejan unas puntuaciones medias que oscilan entre 7.66 y 8.49 , y la desviación estándar ofrece, en todos los casos, valores mayores a 1.40 (dentro de un rango de respuesta entre 0 y 10). Con excepción de los reactivos 1,3 y 7 todos los valores de asimetría y curtosis se encuentran dentro del rango \pm 3.0 por lo que se infiere que las variables se ajustan razonablemente a una 
distribución normal. En cuanto a los índices de discriminación todos los reactivos discriminan satisfactoriamente con índices de discriminación por encima de .30 (Brzoska y Razum, 2010).

\section{Análisis factoriales confirmatorios}

Los resultados globales del análisis factorial confirmatorio en la submuestra 1 (GFI .922; RMSEA .067; CFI .691) y la submuestra 2 (GFI .886; RMSEA .085; CFI .921) para el modelo M1 que corresponde a la distribución original de los reactivos dentro del cuestionario de la Escala de Autoeficacia Académica, indican que el modelo de medición, en ambas submuestras se puede considerar como aceptable aunque no óptimo (Tabla 2).

Tabla 1. Análisis descriptivos e índices de discriminación de los reactivos del cuestionario "Escala de Autoeficacia Académica". Muestra total. Nota: $\mathrm{M}$ = media; DE = desviación estándar; $\mathrm{AS}$ = asimetría; CU $=$ curtosis; ri-total = correlación elemento-total corregida .

\begin{tabular}{|c|c|c|c|c|c|}
\hline \multicolumn{1}{|c|}{ Ítem } & $\mathrm{M}$ & $\mathrm{DE}$ & $\mathrm{AS}$ & $\mathrm{CU}$ & $\mathrm{r}_{\text {i-total }}$ \\
\hline Reactivo 1 & 8.39 & 1.72 & -1.59 & 3.41 & .60 \\
\hline Reactivo 2 & 8.15 & 1.74 & -1.35 & 2.51 & .60 \\
\hline Reactivo 3 & 8.15 & 1.71 & -1.44 & 3.38 & .65 \\
\hline Reactivo 4 & 8.12 & 1.75 & -1.13 & 1.59 & .67 \\
\hline Reactivo 5 & 8.00 & 1.81 & -1.26 & 2.15 & .65 \\
\hline Reactivo 6 & 7.96 & 1.92 & -1.20 & 1.63 & .66 \\
\hline Reactivo 7 & 8.28 & 1.65 & -1.54 & 3.92 & .73 \\
\hline Reactivo 8 & 7.95 & 1.68 & -1.03 & 1.59 & .67 \\
\hline Reactivo 9 & 8.05 & 1.80 & -1.11 & 1.37 & .66 \\
\hline Reactivo 10 & 8.12 & 1.84 & -1.31 & 2.14 & .69 \\
\hline Reactivo 11 & 8.36 & 1.67 & -1.43 & 2.99 & .65 \\
\hline Reactivo 12 & 8.49 & 1.49 & -1.35 & 2.51 & .75 \\
\hline Reactivo 13 & 8.14 & 1.67 & -1.20 & 2.26 & .68 \\
\hline Reactivo 14 & 8.16 & 1.65 & -1.24 & 2.34 & .63 \\
\hline Reactivo 15 & 8.33 & 1.60 & -1.28 & 2.29 & .72 \\
\hline Reactivo 16 & 7.66 & 2.14 & -1.18 & 1.41 & .60 \\
\hline
\end{tabular}

Tabla 2 Índices absolutos, incrementales y de parsimonia para los modelos generados. Submuestras 1 y 2.

\begin{tabular}{|c|c|c|c|c|c|c|c|c|c|}
\hline & \multicolumn{2}{|c|}{ Índices absolutos } & \multicolumn{2}{c|}{ Índices incrementales } & \multicolumn{2}{c|}{ Índices de parsimonia } \\
\hline Modelo & $\chi^{2}$ & GFI & RMSEA & AGFI & TLI & CFI & CMIN/DF & AIC \\
\hline \multicolumn{8}{|c|}{ Primera solución factorial (submuestra 1) } \\
\hline M1 & $248.171^{*}$ & .922 & .067 & .889 & .939 & .951 & 2.585 & 328.171 \\
\hline M1b & $124.363^{*}$ & .946 & .073 & .903 & .945 & .964 & 2.892 & 194.363 \\
\hline \multicolumn{8}{|c|}{ Segunda solución factorial (submuestra 2) } \\
\hline M1 & $346.971^{*}$ & .886 & .085 & .839 & .902 & .921 & 3.614 & 426.971 \\
\hline M1b & $102.078^{*}$ & .953 & .062 & .915 & .961 & .975 & 2.374 & 172.078 \\
\hline
\end{tabular}

En la Tabla 2, * $p<.05 ; \mathrm{GFI}=$ índice de bondad de ajuste; RMSEA = raíz del error medio; AGFI = índice corregido de la bondad de ajuste; TLI=índice de Tucker-Lewis; CFI = índice de ajuste comparativo; CMIN/DF = índice de ajuste chi cuadrado dividido por los grados de libertad; AIC = criterio de información de Akaike

El conjunto de los cuatro factores del modelo M1 explican aproximadamente el $68 \%$ de la varianza en la primer submuestra y el $69 \%$ de la varianza en la segunda submuestra. Por otro lado cuatro de los 16 reactivos, en ambas submuestras, saturan por debajo de .70 en su dimensión prevista ( 1 cumplir con las tareas que se me asignan, 2 Escuchar con atención cuando el profesor aclara una duda a un compañero, 3 Escuchar con atención las preguntas y aportaciones de mis compañeros y 16 Dedicar más horas al estudio de las que había planificado cuando se aproxima un examen). Observándose además, intercorrelaciones altas entre los cuatro factores evidenciando una no muy adecuada validez discriminante entre ellos. 
Los resultados globales del análisis factorial confirmatorio en la primer (GFI .946; RMSEA .073; CFI .964) y segunda submuestra (GFI .953; RMSEA .062; CFI .975), del segundo modelo sometido a prueba (M1b) que corresponde a una estructura tetradimensional del cuestionario sin los reactivos de más baja saturación en cada uno de los factores, indican que este modelo de medición es mejor que el modelo anterior y que su ajuste es óptimo (Tabla 2). Los cuatro factores de este modelo explican en conjunto, en ambas submuestras aproximadamente el $74 \%$ de la varianza. Por otro lado de acuerdo a los resultados de la Tabla 3; ninguno de los 12 reactivos, en ambas submuestras, saturan por debajo de .70 en su dimensión prevista. Observándose además, intercorrelaciones altas entre los cuatro factores evidenciando una no muy adecuada validez discriminante entre ellos.

Tabla 3 Soluciones estandarizadas análisis factorial confirmatorio para el Modelo M1b.

Submuestra 1 y 2. Nota: F1 = Comunicación, F2 = Atención, F3 = Comprensión, F4 = Excelencia

\begin{tabular}{|c|c|c|c|c|c|c|c|c|}
\hline \multirow[b]{2}{*}{ Reactivo } & \multicolumn{4}{|c|}{ Submuestra 1} & \multicolumn{4}{|c|}{ Submuestra 2} \\
\hline & F1 & F2 & F3 & F4 & $\mathrm{F} 1$ & F2 & F3 & F4 \\
\hline \multicolumn{9}{|l|}{ Pesos Factoriales } \\
\hline 4 Expresar mis ideas con claridad & .80 & & & & .79 & & & \\
\hline 5 Hacer comentarios y aportaciones pertinentes & .73 & & & & .72 & & & \\
\hline $\begin{array}{l}6 \text { Hablar o expresarme enfrente de una clase o grupo de } \\
\text { gente }\end{array}$ & .76 & & & & .75 & & & \\
\hline $\begin{array}{l}9 \text { Entablar un diálogo con mis profesores en caso de } \\
\text { desacuerdo con ellos }\end{array}$ & .65 & & & & .74 & & & \\
\hline 7 Poner atención cuando los profesores dan la clase & & .75 & & & & .70 & & \\
\hline 8 Poner atención cuando un compañero expone en clase & & .73 & & & & .62 & & \\
\hline $\begin{array}{l}12 \text { Escuchar con atención las preguntas y comentarios de } \\
\text { mis profesores }\end{array}$ & & .79 & & & & .83 & & \\
\hline 13 Identificar las ideas principales de un texto & & & .81 & & & & .76 & \\
\hline 14 Escribir de manera coherente y organizada & & & .76 & & & & .70 & \\
\hline $\begin{array}{l}15 \text { Relacionar los conceptos nuevos que estoy estudiando } \\
\text { con otros que ya conozco }\end{array}$ & & & .78 & & & & .82 & \\
\hline $\begin{array}{l}10 \text { Preparar mis exámenes apoyándome en los apuntes } \\
\text { de clase, el texto del curso y lecturas adicionales }\end{array}$ & & & & .73 & & & & .70 \\
\hline $\begin{array}{l}11 \text { Entregar puntualmente los trabajos que se me } \\
\text { encargan }\end{array}$ & & & & .76 & & & & .69 \\
\hline \multicolumn{9}{|l|}{ Correlaciones Factoriales } \\
\hline F1 & - & & & & - & & & \\
\hline F2 & .81 & - & & & .88 & - & & \\
\hline F3 & .79 & .86 & - & & .89 & .93 & - & \\
\hline F4 & .76 & .99 & .77 & - & .78 & .99 & .97 & - \\
\hline
\end{tabular}

Invarianza de la estructura factorial entre las submuestras

Los índices de ajuste obtenidos (Tabla 4) permiten aceptar la equivalencia de los modelos de medida básicos entre las dos submuestras. Aunque el valor de Chi-cuadrado excede al exigido para aceptar la hipótesis de invarianza, los índices $\mathrm{GFI}=.950, \mathrm{CFI}=.970$, RMSEA=.048 y $\mathrm{AlC}=366.441$ contradicen esta conclusión lo que nos permite aceptar el modelo base de la invarianza (modelo sin restricciones).

Añadiendo al modelo base restricciones sobre las cargas factoriales caracterizamos la invarianza métrica. Los valores que se recogen en la tabla 4 permiten aceptar este nivel de invarianza. El índice de ajuste general (GFI .946) y el error cuadrático medio de aproximación (RMSEA .047) siguen aportando información convergente en esta dirección. Además, el criterio de información de Akaike (AIC 368.538) y el índice comparativo de Bentler (CFI .967) no sufren grandes variaciones respecto al modelo anterior. Haciendo uso del criterio para la evaluación de los modelos anidados propuesto por Cheung y Rensvold (2002), quiénes sugieren que si el cálculo de la diferencia de los CFI de ambos modelos anidados disminuye en .01 o menos, se da por bueno el modelo restringido y por tanto el cumplimiento de la invarianza factorial; la diferencia entre CFls obtenida permite aceptar el modelo de invarianza métrica. Podemos concluir hasta ahora que las cargas factoriales son equivalentes en las dos submuestras.

Una vez demostrada la invarianza métrica entre las submuestras, pasamos a evaluar la equivalencia entre interceptos (invarianza factorial fuerte). Los índices (Tabla 4) muestran un ajuste aceptable de este modelo, tanto evaluado de modo independiente como analizándolo respecto a su anidamiento con el modelo de 
invarianza métrica. La diferencia entre los índices comparativos de Bentler es de .003; el índice de ajuste general es .946 y el error cuadrático medio de aproximación es .048. Aceptada la invarianza fuerte, los dos modelos evaluados son equivalentes respecto a los coeficientes factoriales y a los interceptos. En la Tabla 4, Nota: * $\mathrm{p}<.05 ; \mathrm{GFI}=$ índice de bondad de ajuste; $\mathrm{NFI}=$ índice de ajuste normado; $\mathrm{CFI}=$ índice de ajuste comparativo; RMSEA = raíz del error medio; AIC = criterio de Información de Akaike

Los factores obtenidos en los análisis factoriales confirmatorios alcanzan, en su mayoría valores de consistencia interna por encima de .75 en ambas submuestras evidenciando una consistencia interna adecuada para este tipo de subescalas, particularmente si se considera el número reducido de reactivos (Tabla 5).

Tabla 4 Índices de bondad de ajuste de cada uno de los modelos puestos a prueba en la invarianza factorial.

\begin{tabular}{|l|c|c|c|c|c|c|c|}
\hline \multicolumn{1}{|c|}{ Modelo } & \multicolumn{7}{c|}{ Índice de Ajuste } \\
\hline & $\chi^{2}$ & gl & GFI & NFI & CFI & RMSEA & AIC \\
\hline Modelo sin restricciones & $226.441^{*}$ & 86 & .950 & .952 & .970 & .048 & 366.441 \\
\hline Invarianza métrica & $244.538^{*}$ & 94 & .946 & .948 & .967 & .047 & 368.538 \\
\hline Invarianza factorial fuerte & $272.991^{*}$ & 104 & .946 & .942 & .963 & .048 & 376.991 \\
\hline
\end{tabular}

Tabla 5. Coeficiente omega y alfa para los factores obtenidos en los análisis factoriales exploratorios submuestras 1 y 2 .

\begin{tabular}{|l|c|c|c|c|}
\hline & \multicolumn{2}{|c|}{ Submuestra 1 } & \multicolumn{2}{c|}{ Submuestra 2 } \\
\hline \multicolumn{1}{|c|}{ Factor } & $\Omega$ & $\alpha$ & $\Omega$ & $\alpha$ \\
\hline Comunicación & .83 & .82 & .84 & .85 \\
\hline Atención & .80 & .79 & .76 & .84 \\
\hline Comprensión & .83 & .80 & .80 & .82 \\
\hline Excelencia & .71 & .68 & .65 & .72 \\
\hline
\end{tabular}

\section{DISCUSIÓN}

El objetivo principal del estudio fue indagar si se replican o no los resultados psicométricos propuestos por Rangel (2015) para la Escala de Autoeficacia Académica a través de una muestra de universitarios utilizando el análisis factorial confirmatorio (AFC). Los análisis factoriales confirmatorios realizados en cada submuestra por separado apoyan la estructura factorial de cuatro factores: Comprensión, Comunicación, Atención y Excelencia obtenida por Rangel (2015) al evidenciar una consistencia interna adecuada, particularmente si se considera el número reducido de reactivos en cada uno de ellos; al mismo tiempo que los factores así obtenidos presentan en general saturaciones factoriales estandarizadas adecuadas, saturaciones que se corresponden con las halladas en el estudio de Rangel (2015); sin embargo hubo que eliminar cuatro reactivos ( 1 cumplir con las tareas que se me asignan, 2 Escuchar con atención cuando el profesor aclara una duda a un compañero, 3 Escuchar con atención las preguntas y aportaciones de mis compañeros y 16 Dedicar más horas al estudio de las que había planificado; cuando se aproxima un examen). Sugiriendo además la existencia de fuertes evidencias de la validación cruzada de la medida y por tanto de la estabilidad de la estructura hasta que no se demuestre lo contrario.

En síntesis, el análisis de las propiedades psicométricas de la Escala de Autoeficacia Académica, ha mostrado, tanto en este estudio como en el llevado a cabo por Rangel (2015), que una estructura tetrafactorial es viable y adecuada de acuerdo a los requisitos psicométricos establecidos cuando los informantes son los propios alumnos. La estructura de cuatro factores, atendiendo a criterios estadísticos y sustantivos, ha mostrado adecuados indicadores de ajuste, de fiabilidad y de validez. Sin embargo, el alcance de estos resultados es limitado, y es necesario que en investigaciones futuras se confirme la estructura obtenida, lo cual permitirá contar con evidencia más robusta respecto a la estructura factorial de la escala. Específicamente, debe demostrarse si la invarianza de la estructura de la escala se cumple por género, edad, entre alumnos de distintas licenciaturas, entre otras; de tal manera que, se considera que más estudios son necesarios con el fin de corroborar o refutar los datos obtenidos en las investigaciones realizadas hasta el momento. Asimismo, es indispensable comprobar si la escala resulta útil para estudiar la relación entre autoeficacia académica y aprendizaje. 


\section{CONCLUSIONES}

Con base en el estudio presentado, se pueden extraer las siguientes con conclusiones principales:

i) El Análisis Factorial Confirmatorio, en ambas muestras, indicó que el ajuste de los datos al modelo teórico de 12 reactivos agrupados en cuatro factores es aceptable.

ii) Los factores en ambas muestras evidenciaron una consistencia interna adecuada, particularmente si se considera el número reducido de reactivos en cada uno de ellos

iii) Los resultados del análisis de la invarianza factorial entre las muestras; indican una alta congruencia entre pares de factores.

\section{REFERENCIAS}

Abalo J., Lévy J., Rial A. y Varela J., Invarianza factorial con muestras múltiples, En Modelización con Estructuras de Covarianzas en Ciencias Sociales por J. Lévy, pp 259-278 Netbiblo, Madrid, (2006)

Arbuckle J. R., AMOS users guide version 21.0, Marketing Department, SPSS Incorporated, Chicago, IL, (2012)

Bandura A., Self-efficacy mechanism inhuman agency, American Psychologist, 37(2), 122-147, (1982)

Bandura A., Social foundations of thought and action: A social cognitive theory, Prentice Hall, Englewood Cliffs, NJ, (1986)

Bandura A., Self-efficacy: The exercise of Control, Freeman, New York, (1997)

Bandura A., On the Functional Properties of Perceived Self-Efficacy Revisited, doi: 10.1177/0149206311410606, Journal of Management, 38(1), 9-44, (2012)

Beaudoin M. y Desrichard O., Are Memory Self-Efficacy and Memory Performance Related? A MetaAnalysis, Psychological Bulletin, 137(2), 211-241, (2011)

Blanco Á., Creencias de autoeficacia de estudiantes universitarios: un estudio empírico sobre la especificidad del constructo, RELIEVE, 16(1), 1-28, (2010)

Blanco H., Ornelas M., Tristán J. L., Cocca A., Mayorga-Vega D., López-Walle J., et al., Editor for creating and applying computerise surveys, doi: http://dx.doi.org/10.1016/j.sbspro.2013.12.105, Procedia Social and Behavioral Sciences, 106, 935-940, (2013)

Brzoska P. y Razum O., Validity Issues in Quantitative Migrant Health Research: The Example of IIIness Perceptions, Peter Lang International Academic Publishers, New York, NY, (2010)

Byrne B. M., Structural Equation Modeling With AMOS: Basic Concepts, Applications, and Programming, Routledge, New York, NY, (2010)

Cheung G. W. y Rensvold R. B., Evaluating goodness-of-fit indexes for testing measurement invariance, doi: 10.1207/s15328007SEM0902_5, Structural Equation Modeling, 9(2), 233-255, (2002)

Elosua P. y Zumbo B. D., Coeficientes de fiabilidad para escalas de respuesta categórica ordenadas, Psicothema, 20(4), 896-901, (2008)

Gelabert E., García-Esteve L., Martín-Santos R., Gutiérrez F., Torres A. y Subirà S., Psychometric properties of the Spanish version of the Frost Multidimensional Perfectionism Scale in women, Psicothema, 23(1), 133139, (2011)

Hannah S. T., Avolio B. J., Walumbwa F. O. y Chan A., Leader Self and Means Efficacy: A multi-component approach, doi: 10.1016/j.obhdp.2012.03.007 Organizational Behavior and Human Decision Processes, 118(2), 143-161, (2012)

Hasheminasab M., Ghanbari Z., Azizi J. y Shamsi M., Investigating the Relationship between Self-Efficacy with Academic Achievement, Discipline, Urban-Rural and Order Birth of High School Students in Rafsanjan, International Journal of Psychology and Behavioral Research, 3(4), 258-264, (2014)

Javanmard A., Hoshmandja M. y Ahmadzade L., Investigating the relationship between self-efficacy, Cognitive and metacognitive strategies, and academic self-handicapping with academic achievement in male high school students in the Tribes of Fars Province, Journal of life Science and Biomedicine, 3(1), 27-34, (2012) 
Montero I. y León O., Sistema de clasificación del método en los informes de investigación en Psicología, International Journal of Clinical and Health Psychology, 5, 115-127, (2005)

Nunnally J. C. y Bernstein I. H., Teoría Psicométrica, McGraw-Hill, México, (1995)

Peguero A. A. y Shaffer K. A., Academic Self-Efficacy, Dropping Out, and the Significance of Inequality, doi: 10.1080/02732173.2014.978428, Sociological Spectrum, 35(1), 46-46, (2015)

Pérez E., Lescano C., Heredia D., Zalazar P., Furlám L. y Martínez M., Desarrollo y análisis psicométricos de un inventario de autoeficacia para inteligencias múltiples en niños argentinos Psicoperspectivas, 10(1), 169-189, (2011)

Rangel Y. S., Validación de una escala de autoeficacia académica: análisis de un modelo mediante ecuaciones estructurales, Tesis doctoral, Universidad de Chihuahua, México, (2015)

Revelle W. y Zinbarg R. E., Coefficients alpha, beta, omega and the glb: comments on Sijtsma, doi: 10.1007/s11336-008-9102-z, Psychometrika, 74(1), 145-154, (2009)

Richardson M., Abraham C. y Bond R., Psychological correlates of university students' academic performance: a systematic review and meta-analysis, doi: 10.1037/a0026838, Psychological Bulletin, 138(2), 353-387, (2012)

Robbins S. B., Lauver K., Le H., Davis D., Langley R. y Carlstrom A., Do psychosocial and study skill factors predict college outcomes? A meta-analysis, doi: 10.1037/0033-2909.130.2.261, Psychological Bulletin, 130(2), 261-288, (2004)

Rodríguez M. N., Análisis factorial confirmatorio de la versión uruguaya de la escala Smart de Trapnell para medir capacidad intelectual percibida, Revista Iberoamericana de Diagnóstico y Evaluación Psicológica, 1(27), 85-105, (2009)

Shkullaku R., The relationship between self - efficacy and academic performance in the context of gender among Albanian students, European Academic Research, 1(4), 467-478, (2013)

Sijtsma K., On the use, the misuse, and the very limited usefulness of Cronbach's alpha, doi: 10.1007/s11336-008-9101-0, Psychometrika, 74(1), 107-120, (2009)

Thompson B., Exploratory and Confirmatory Factor Analysis. Understanding concepts and applications. , American Psychological Association, Washington, D C, (2004)

Yeo G. B. y Neal A., An examination of dynamic relationship between self-efficacy and performance across levels of analysis and levels of specificity, Journal of Applied Psychology, 91(5), 1088-1101, (2006) 